\title{
CONTRIBUCIÓN AL ESTUDIO HISTORIOGRÁFICO DE LA TRADUCCIÓN. PROPUESTA DE UN MANUAL DE LECTURAS GUIADAS Y SUS APLICACIONES
}

\author{
Pilar Ordóñez López \\ mordonez@trad.uji.es \\ Universitat Jaume I \\ José Antonio Sabio Pinilla \\ jasabio@ugr.es \\ Universidad de Granada
}

\section{Resumen}

Tras el auge experimentado en los últimos años por las antologías que recogen el discurso de la traducción a lo largo del tiempo, parece llegado el momento de reflexionar sobre el tipo de investigación histórica que se está haciendo en nuestros días. En esta línea, defendemos en este artículo la necesidad de elaborar un manual de lecturas guiadas de historiografía de la traducción que proporcione algunas claves para entender la pluralidad y el dinamismo de nuestra disciplina. Dentro de la tradición del reader, la obra cumpliría una función eminentemente pedagógica e incluiría una muestra representativa y bien contextualizada de la investigación producida en el campo histórico de la traducción en los últimos cuarenta años.

\begin{abstract}
"Towards a Historiographical Study of Translation. A Proposal for a Historiography Reader and its Applications"

Following an increase in the publication of anthologies focusing on the diachronic dimension of translation discourse in recent years, it seems timely to consider the historical research that has been carried out in this area in the last decades. The present article argues that there is a need to compile a translation historiography reader that serves to provide some keys to understanding the evolution of this multi-faceted
\end{abstract}


and dynamic discipline. In line with the traditional remit of this genre, the proposed reader will have a mainly pedagogical function and will contain a well-contextualised selection of texts representative of research in the area of the History of Translation over the past forty years.

Palabras clave: Historiografía de la traducción. Historia de la traducción. Teoría de la traducción. Manual de lecturas guiadas. Antologías.

Keywords: Translation Historiography. Translation History. Theory of Translation. Reader. Anthologies.

Manuscript received on June 26, 2012 and accepted on September 20, 2012. 
En este artículo defendemos la necesidad de elaborar un manual de lecturas guiadas de historiografía de la traducción una vez que el género de la antología cuenta ya, dentro de los estudios históricos de la traducción, con un buen número de obras que rescatan los textos del pasado en diferentes lenguas y tradiciones. Así pues, consideramos llegada la hora de organizar una obra que recoja una muestra amplia y representativa de la investigación historiográfica realizada en el campo de la traducción durante los últimos cuarenta años. Pensamos que un volumen dedicado al estudio de la investigación histórica y de las teorías de la traducción puede proporcionar algunas claves para entender la evolución de nuestra disciplina. El estudio historiográfico permitiría reflexionar sobre el modo como hemos afrontado esa evolución y, en concreto, podría ayudar a evitar dos riesgos muy extendidos, como son que el estudio del pasado se haga por el historiador con las ideas del presente y que la explicación de las prácticas traductoras del pasado se haga de manera aislada (cf. D’hulst 1991, 2001; López García 2011; Fernández Sánchez 2012). Por otra parte, el manual que proponemos permitiría mostrar la pluralidad de enfoques traductológicos que, desde diferentes tradiciones, abordan el estudio de la disciplina. En este sentido, la propuesta que aquí presentamos quiere reflejar esta diversidad actual en la investigación historiográfica de la traducción, así como subrayar la conveniencia de la internacionalización de la misma con objeto de favorecer un pensamiento no eurocéntrico (cf. Tymozcko 2007: 15-53). Por último, pero no menos importante, debemos recordar la función didáctica de esta obra, que va dirigida al ámbito universitario y pretende tener un valor instrumental dentro del aula como apoyo a las asignaturas de teoría e historia de la traducción.

\section{La investigación historiográfica en traducción: diversidad de enfoques y pertinencia de su estudio}

Distinguimos como Lambert (1993: 4) entre historia e historiografía: la primera haría referencia fundamentalmente al material histórico mientras que la segunda abarcaría el ámbito del discurso del historiador. Por su parte, Delisle (2008: 82) menciona tres acepciones de historiografía: a) el arte de escribir 
la historia; b) el conjunto de obras históricas producidas por una época determinada o por una determinada disciplina, en nuestro caso, los estudios de traducción; y c) la mirada histórica sobre esta producción e interesada por la evolución de los métodos de investigación del historiador y los modos de escribir la historia. Por último, D’hulst (2010: 397-398), establece tres niveles o subdisciplinas: la historia, entendida como "the proper sequence of facts, events, ideas, discourses, etc."; la historiografía, definida como "la historia de las historias", en la que se combinan conceptos y metodología históricos y métodos específicos de otras áreas de conocimiento como la lingüística, la filosofía o la literatura en el análisis de la investigación histórica propiamente dicha; y la metahistoriografía, que hace referencia a la reflexión explícita sobre los conceptos y métodos para escribir la historia, así como a los problemas epistemológicos y metodológicos relacionados con dichos conceptos y métodos y que tienen que ver con cuestiones espaciales, temporales, ideológicas y con el acceso a las fuentes y su interpretación.

Las teorías de la traducción son resultado de una historicidad compleja, evolucionan y reflejan problemas pasados, que se repiten de nuevos modos, por lo que las teorías conviven unas con otras, se influyen mutuamente y se perfilan (cf. Pym 2010). Autores como Lambert, Hermans, D'hulst, Delisle, Pym, Bastin o Badia, por citar algunos nombres, han señalado la necesidad de estudiar la historia y sus fundamentos. Recordaremos que en la división de los Estudios de Traducción propuesta por Holmes (1972, 1988) no aparecía un apartado específico dedicado a la historia de la traducción. Sin embargo, al tratar de los estudios descriptivos, orientados al producto (la descripción y comparación de traducciones en un plano sincrónico y diacrónico) y a la función (la descripción de la función de la traducción en la situación sociocultural), se refería a la variante diacrónica, lo que nos llevaría en el producto a una "general history of translation - however ambitious such a goal may sound at this time", y en la función nos llevaría a ver la influencia "in histories of translations and in literary histories" (1988: 72). Al final de su artículo llamaba la atención sobre la relación dialéctica que mantienen las tres ramas, en las cuales habría que incorporar dos dimensiones de análisis: la histórica y la metodológica (o metateórica):

[...] in each of the three branches of translation studies, there are two further dimensions that I have not mentioned, dimensions having to do with the study, not of translating and translations, but of translation studies itself. One of these dimensions is historical: there is a field of the history of translation theory, in which some valuable work has been done, but also one of the history of translation description and of applied translation studies (largely a history of translation teaching and translator training) both of which are 
fairly well virgin territory. Likewise there is a dimension that might be called the methodological or meta-theoretical, concerning itself with problems of what methods and models can best be used in research in the various branches of the discipline (how translation theories, for instance, can be formed for greatest validity, or what analytic methods can best be used to achieve the most objective and meaningful descriptive results), but also devoting its attention to such basic issues as what the discipline itself comprises (cf. Holmes 1988: 79).

Esa perspectiva vendría de la mano de autores como Toury, Hermans, Lefevere o Lambert, quienes desde los postulados de la Teoría de los Polisistemas contribuyeron al desarrollo de los Estudios Descriptivos de Traducción (cf. Lambert 1995) e introdujeron los conceptos de norma, manipulación o reescritura. De este modo, se han ido incorporando diferentes cuestiones al estudio histórico de la traducción, tales como la ideología, la manipulación y el poder, o los agentes de la traducción, sobre todo los traductores, como parte de los últimos paradigmas asociados a los Cultural and ideological turns (Susan Bassnett, Lawrence Venuti, Maria Tymoczko, Edwin Gentzler), incluidos los enfoques postcoloniales y de género, además de los sociológicos e incluso filosóficos, campos donde el pasado y el presente adquieren nuevas relaciones dentro de un marco cada vez más global (como defienden Cronin 2003 y Tymoczko 2007). Al mismo tiempo, los análisis principalmente descriptivos que caracterizan las últimas décadas del siglo $\mathrm{XX}$ van dando paso a estudios de naturaleza interpretativa (cf. Bastin $\&$ Bandia 2006: 2), con el desarrollo de una metodología basada en la historiografía de la traducción. La incorporación de este componente interpretativo ha fomentado, a su vez, un mayor reconocimiento de la interrelación e imbricación de la historia de la traducción con otras áreas de conocimiento, caso de la sociología, la historia o la literatura comparada, en las que, como declara Santoyo (2009: 489), la traducción suele —o solía- estar ausente.

En suma, desde el cambio de perspectiva teórica provocado por los polisistemas, cuya influencia fue decisiva para la evolución de los estudios descriptivos de la traducción al incluir las traducciones como obras propias del sistema receptor e incidir en el carácter histórico de la actividad, a lo que hay que añadir el interés por las cuestiones metodológicas inspiradas en el trabajo del historiador (cf. Delisle 1997-98; Pym 1992, 1998) y, más recientemente aún, el giro dado por los enfoques historiográficos posmodernos que llaman la atención sobre el estudio plural del pasado desde una visión no canónica y marcada por la ideología de quien escribe la historia (cf. Bandia 2006; Fernández Sánchez 2010), la investigación histórica ha recobrado un nuevo interés y se ha convertido en objeto de discusión. Así, en las últimas aproximaciones 
se distingue el pasado (la realidad histórica) de la historia (entendida como interpretación narrativa) lo que supone que "el conocimiento histórico no se relacione únicamente con una serie de contenidos, sino también con prácticas discursivas determinadas culturalmente" (cf. Fernández Sánchez 2010: 232).

En el ámbito académico, el estudio del pasado de la disciplina se aborda desde múltiples perspectivas en función de la formación de los investigadores y de sus tradiciones traductológicas, y esto se hace en torno a un objeto de estudio caracterizado por una considerable heterogeneidad en la que, además, en los últimos años, se observa una expansión que va más allá de los aspectos puramente traductológicos. En la investigación historiográfica moderna encontramos una serie de propuestas que, como la presentada por D'hulst (2001: 21-32) siguiendo el esquema de la retórica clásica, sirven para clasificar los objetos de la historiografía y reflejan la potencialidad de la investigación teórica e histórica de los Estudios de Traducción:

- Quis?, quiénes son los traductores, cómo se han formado como individuos o como grupo.

- Quid?, qué tipo de obras se han traducido.

- Ubi?, dónde se han realizado las traducciones, dónde se han publicado.

- Quibus auxiliis?, mecenazgo, marco ideológico, etc.

- Cur?, por qué se producen, qué consecuencias, qué tipo de relaciones establecen con los textos originales, etc.

- Quando?, cuestiones de periodización.

- Cui bono?, función social de las traducciones, recepción y uso, etc.

Por ello, se hace necesario reflexionar sobre los métodos que los investigadores han propuesto para analizar y estudiar el pasado de la disciplina, no solo atendiendo a los hechos, fuentes y datos, sino también a cómo se han planteado las necesidades de su estudio y se han enfocado los problemas que ese estudio presenta. Hoy día, como señalan Pym (2010), D'hulst (2011) o Munday (2012), hay que hacerse aún muchas preguntas relacionadas con algunos conceptos fundamentales para el estudio de la teoría e historia de la traducción. Estas preguntas tienen que ver con el lugar y la función de la historia en los estudios de traducción o con la relación de la investigación histórica con la literatura comparada y las historias literarias nacionales, así como con los objetos propios del estudio histórico y la aplicación de las teorías. En definitiva, nos enfrentan al análisis del papel que ejercen las cuestiones teóricas e históricas en la reflexión actual de la disciplina al tiempo que plantean la necesidad de reflexionar sobre la pertinencia de los modelos que aplicamos al estudio de la traducción. 
Todas estas cuestiones nos llevan a observar la variedad y complejidad que encierra el estudio de la historia y que un manual de lecturas guiadas, convenientemente ideado, puede plantear de modo directo a los estudiosos y estudiantes de traducción. Además, una obra así ayudaría a mostrar la pluralidad de enfoques teóricos desde los cuales se afronta el estudio del pasado y que sitúan al historiador de la traducción en una posición privilegiada pues su tarea resulta, precisamente por esa diversidad, más decisiva (cf. Hermans 1999: 101).

\section{Pensamiento traductor y antologías sobre la traducción. Un manual de lecturas guiadas}

Las antologías sobre la traducción desempeñan un papel fundamental en el estudio y la reconstrucción de la historia de la reflexión en torno a la traducción. Son obras que recopilan textos teóricos generalmente acompañados de un extenso prólogo explicativo en el que se explicitan los criterios de selección aplicados en la recopilación. Pretenden reunir y poner al alcance del lector los textos más representativos de la reflexión traductora a lo largo de la historia; en su mayoría, se trata de textos de naturaleza secundaria y muy variada — cartas, prólogos, prefacios o introducciones-y, por ello, de difícil acceso.

Si las antologías literarias son resultado de una concepción determinada de la historia literaria, en las antologías sobre la traducción subyace, de igual modo, una visión de la historia de la traducción. A las características expuestas anteriormente, hay que añadir además el papel de las antologías en la formación, desarrollo y consolidación del canon. La selección efectuada por el antólogo en su compilación no responde meramente a la intención de recopilar un fragmento del pasado sino que también, como argumenta Guillén ([1985] 2005: 378) en el ámbito literario, "practica una idea de la literariedad, fijando géneros, destacando modelos, afectando el presente del lector, y sobre todo, orientándole hacia un futuro". Existe, pues, una doble intencionalidad subyacente en la recopilación llevada a cabo por el antólogo en la que se combinan pasado, presente y futuro, lo cual convierte a las antologías en "autoridades capaces de conformar cánones" (cf. Enríquez Aranda 2007: 123).

Dentro de los estudios históricos de la traducción observamos un creciente interés por el género de la antología, desde la primera de Störig (1963) hasta nuestros días, con incidencia en diversos ámbitos culturales y especialmente patente en el ámbito de la Península Ibérica, donde encontramos quince antologías aparecidas entre 1987 y 2011 (cf. Sabio Pinilla \& Ordóñez López 2012). Este conjunto de antologías pone de relieve el hecho de que se 
ha empezado el proceso de recopilación de los textos teóricos clásicos — tanto en sentido cronológico como descriptivo- de la traducción, textos en los que vamos a encontrar cuestiones muy parecidas a las que actualmente ocupan las páginas de las publicaciones sobre Traductología, si bien, claro está, planteadas en su mayoría desde otros puntos de vista por un conjunto heterogéneo de teóricos y estudiosos que tuvieron contacto, ya sea como traductores o bien como lectores de traducciones, con la actividad traductora.

Si las antologías que recogen las reflexiones sobre la traducción a lo largo del tiempo gozan de una enorme vitalidad en el campo de los estudios de traducción, especialmente en el ámbito peninsular, no ocurre lo mismo con la modalidad del manual de lecturas guiadas que aquí presentamos. Esta variedad, aún poco explorada en los estudios peninsulares de la traducción y que se inspira en el modelo anglosajón del reader, ${ }^{1}$ es la que describimos a continuación como paso previo de nuestra propuesta.

\subsection{Un modelo anglosajón: el reader}

Al hablar de las antologías sobre la traducción hemos de tener en cuenta los denominados readers, que se definen como "books of collected or assorted writings, esp. when related in theme, autorship, or instructive purpose; anthology". ${ }^{2}$ Si bien anthology y reader parecen ser sinónimos, ${ }^{3}$ en el ámbito traductológico anglosajón hallamos con mayor frecuencia el término reader, o variantes como readings o critical readings, denominaciones todas ellas que subrayan su función pedagógica, que como en el caso de las antologías (cf. Sabio Pinilla \& Ordóñez López 2012: 98) constituye, a su vez, una de las principales motivaciones para la elaboración de este tipo de trabajos.

En esencia, el reader comparte los rasgos definitorios de las antologías; no obstante, y de nuevo ciñéndonos al ámbito traductológico exclusivamente, observamos algunos matices específicos del modelo anglosajón: a) la función didáctica es más acusada; b) por consiguiente, el aparato explicativo es mayor que en las antologías tradicionales; c) la mayoría de los textos seleccionados son de autores contemporáneos, y d) los textos suelen recopilarse de manera íntegra.

1. Adaptación del equivalente "manual de lecturas recomendadas" que propone Antonio Lozano para reader en su diccionario especializado en Biblioteconomía y Documentación, véase eubdl.ugr.es English <> Spanish Dictionary (Granada University, Spain).

2. http://dictionary.reference.com

3. http://www.britannica.com 
Como señalábamos con relación a las antologías, y puede apreciarse en el inventario que aparece más abajo, la publicación de readers también ha experimentado una progresión significativa a partir de la última década. Si hasta los años noventa solo contábamos con el trabajo de Chesterman (1989), actualmente encontramos al menos cinco obras en el campo de los estudios de traducción. A esta categoría pertenecen los siguientes trabajos:

- Chesterman, Andrew (ed.) (1989) Readings in Translation Theory. Helsinki: Oy Finn Lectura Ab.

- Venuti, Lawrence (ed.) (2000) The Translation Studies Reader. London $\&$ New York: Routledge.

- Venuti, Lawrence (ed.) (2004) The Translation Studies Reader. London $\&$ New York: Routledge [2a edición revisada].

- Weissbort, Daniel \& Astradur Eysteinsson (eds.) (2006) Translation. Theory and Practice. A Historical Reader. New York: Oxford University Press.

- Baker, Mona (ed.) (2009) Critical Readings in Translation Studies. London \& New York: Routledge.

Como decíamos, se trata de obras publicadas en la tradición anglosajona y en las que el énfasis recae sobre todo en textos teóricos de investigadores contemporáneos. En este sentido, podemos considerar que los readers son un complemento de las antologías, pues si estas, por lo general, se dedican al estudio de los discursos sobre la traducción en el pasado, los readers recogen textos que, desde diferentes perspectivas, ilustran la evolución de los estudios de traducción como disciplina. Del mismo modo, atendiendo al ámbito o tradición en la que surgen los trabajos, el fenómeno de las antologías se manifiesta especialmente en tradiciones no anglófonas, ${ }^{4}$ en las que, como vemos, se opta por la categoría del reader. Dentro de estas obras hallamos un reader de naturaleza histórica, pues incluye textos no contemporáneos, elaborado por Weissbort y Eysteinsson (2006), y que se titula Historical Reader.

\subsection{Características de los readers}

Readers y antologías se sostienen indudablemente sobre una base común. De este modo, la revisión de los rasgos definitorios del género antológico llevada a cabo en Sabio Pinilla y Ordóñez López (2012: 94-97), partiendo de las

4. Para una relación exhaustiva de las antologías sobre la traducción, véase Sabio Pinilla y Ordóñez López (2012: 102-109). 
propuestas de Guillén ([1985] 2005) y Fraisse (1997), es también aplicable al reader. Si nos situamos en el marco de los estudios de traducción, podemos apreciar que readers y antologías coinciden en el propósito de reunir y poner al alcance del lector una colección de textos que se consideran representativos o influyentes de un determinado periodo, enfoque o tradición.

A continuación, tomando como referencia el análisis realizado por Sabio Pinilla y Ordóñez López (2012: 110-117), repasamos los aspectos más característicos de ambas formas antológicas.

\subsubsection{Reescritura y selección}

Toda antología implica la reescritura de textos ya existentes. No abundaremos aquí en el concepto de reescritura, ${ }^{5}$ pero lo que sí hemos de tener presente, puesto que está íntimamente relacionado con la naturaleza del género antológico, es la vinculación ineludible entre los conceptos de reescritura, ideología y manipulación. Es decir, en estas obras subyace un determinado posicionamiento teórico o una intención que mueve al antólogo y que condicionará su selección de textos, en primer término, así como la presentación, contextualización u ordenación posterior de los mismos.

En el caso del reader, podemos observar una notable variedad en las motivaciones de los autores, en las que se aprecia una evolución que va de la mano del desarrollo experimentado por los estudios de la traducción y que ha permitido ir ampliando la variedad temática y disciplinar de los textos seleccionados, dando cabida a cuestiones tales como la ideología, los estudios de género, la relación entre traducción y poder, además de las aportaciones provenientes de otras disciplinas como la literatura, sociología o lingüística. De este modo si el trabajo de Chesterman (1989) pretende:

to illustrate something of the general development of translation theory towards an increasing concern with textual and pragmatic issues, the potential of machine translation, and cognitive aspects of translation (Chesterman 1989: 6),

entre las motivaciones de Venuti (2000) se incluyen:

to challenge any disciplinary complacency, to produce a consolidation that interrogates the ways in which translation is currently researched and taught $[\ldots]$, to show what translation studies have been and to suggest what they might be (Venuti 2000: 1-2).

5. Autores como Lefevere (1992) y Vidal Claramonte (1998) estudian el concepto de reescritura desde la perspectiva de los estudios de traducción. 
Baker, por su parte, considera que la disciplina ha alcanzado ya una fase de consolidación desde la que podemos movernos con confianza para explorar enfoques más innovadores. Por ello, adopta una perspectiva alejada de dicotomías y taxonomías y afronta la reescritura desde una orientación "deliberately prospective rather than retrospective" (2009: 1) a fin de ayudarnos "to move, to explore new ground, rather than pay tribute to and consolidate past achivements" (2009: 1), al tiempo que trata de incorporar la reflexión surgida en áreas con mayor tradición como la antropología, la literatura o la sociología (2009: 2). Weissbort y Eysteinsson (2006), a su vez, enfocan su reescritura con el propósito de "to illuminate the essential activity of translation from a number of perspectives: historical and contemporary, theoretical and practical" (2006: v). Como es sabido, una de las críticas más frecuentes a la formación de traductores es la falta de vinculación entre teoría y práctica, y es precisamente este vínculo el que pretenden reforzar Weissbort y Eysteinsson, quienes rechazan la posibilidad de limitar su selección a un conjunto de reflexiones canónicas de naturaleza teórica al entender que "it is [...] the practice of translation, which opens the gateway between the present and history" (2006: v). Así, se proponen "to bring across to our readers how valuable reflections about translation took form in contexts of actual translation practice" (2006: v), lo cual les lleva a subrayar esta conexión, de manera más o menos explícita, en toda su selección.

La selección de los textos, estrechamente ligada a las motivaciones que impulsaron la labor de reescritura, desempeña un papel fundamental. En general, el principal criterio de selección es la representatividad de los textos (cf. Chesterman 1989), si bien esta representatividad ha de ser entendida en términos relativos, es decir, en función de la perspectiva u orientación adoptada por el antólogo. Por ejemplo, en la selección ofrecida por Baker el énfasis recae sobre textos, que no solo proceden del ámbito traductológico sino también de otras áreas, que son clave para explorar una serie de temas que la compiladora considera esenciales para el avance de la disciplina (2009: 1).

En relación con el periodo abarcado, la mayoría de los readers se centran en textos de teóricos contemporáneos; Weissbort y Eysteinsson (2006) optan por ofrecer una perspectiva histórica e incluyen también reflexiones clásicas (en un primer bloque que va desde la Antigüedad hasta el siglo XX) y Venuti (2004) dedica un apartado a las que denomina "Foundational Statements", anteriores al siglo $\mathrm{XX}$.

El número de textos seleccionados varía considerablemente de un reader a otro, pero en términos generales el volumen es más reducido que en las 
antologías. Todos los readers son monolingües, esto es, todos los textos recopilados aparecen en inglés tras haberse traducido las aportaciones en otras lenguas.

La heterogeneidad de propósitos o motivaciones que han impulsado los readers hacen que no sea fácil identificar un canon general en cuanto a los autores seleccionados; no obstante, los textos responden a un canon occidental. Por lo que respecta al periodo clásico o fundacional, autores como Dryden y Benjamin aparecen en los trabajos que incorporan reflexiones históricas (cf. Chesterman 1989; Venuti 2004; Weissbort \& Eysteinsson 2006). Vinay y Darbelnet, Jakobson, Nida o Reiss parecen ser imprescindibles en aquellos trabajos en los que se pretende dar una visión general y representativa de la reflexión traductológica contemporánea (cf. Chesterman 1989; Venuti 2000, 2004; Weissbort \& Eysteinsson 2006).

\subsubsection{El reader como libro de lecturas}

El reader, igual que la antología, es el resultado de un proceso de reescritura de textos preexistentes a través del cual surge una nueva obra o, lo que es lo mismo, un libro independiente formado por lecturas guiadas.

Como en el caso de las antologías, los autores de los readers son profesores universitarios de traducción o áreas afines; el reader constituye una herramienta útil para dar a conocer a los estudiantes los textos más influyentes de la reflexión teórica contemporánea o, como hemos visto, de familiarizarlos con las tendencias emergentes en la literatura traductológica.

El aparato paratextual que presentan es uno de los elementos más característicos del reader que, como señala Ruiz Casanova (2007: 162), contribuye a perfilar la autoría del antólogo y a construir el libro como tal. Forman parte de este aparato los prólogos, la organización de los textos, las notas y los comentarios, las lecturas complementarias, la bibliografía y los índices, además de los agradecimientos. Los prólogos contienen la explicación de la motivación y el propósito de la obra, la exposición de los criterios de selección, la justificación ante posibles omisiones, la presentación de la obra y la justificación de la organización de los textos e incluso sugerencias sobre las posibles aplicaciones didácticas del reader (cf. Venuti 2000, 2004). Otro de los componentes fundamentales del aparato paratextual, que resalta aún más la función didáctica de este tipo de obras, es la contextualización o introducción a cada texto (cf. Chesterman 1989), bloque temático (cf. Baker 2009) o cronológico (cf. Venuti 2000, 2004; Weissbort \& Eysteinsson 2006), donde se presenta al autor y se exponen las ideas principales del texto, relacionándolas con otras aportaciones del momento. Esta contextualización es poco frecuente en el 
caso de las antologías tradicionales; de hecho, su ausencia ha motivado una de las críticas más comunes, a saber, la descontextualización de los textos, que puede conducir a una mera historiografía acumulativa y lineal (cf. D'hulst 1991, 1995; Pym 1992, 1998; Lépinette 1997, 2006). Asimismo, refuerzan la función didáctica del reader las sugerencias de lecturas complementarias (cf. Chesterman 1989; Venuti 2000, 2004; Baker 2009) que se proporcionan al final de cada bloque introductorio o de la presentación de los textos y que, de nuevo, no encontramos en el caso de las antologías sobre la traducción.

En relación con la ordenación de los textos, uno de los readers (cf. Baker 2009) los agrupa en secciones temáticas; el resto adopta, simplemente, criterios cronológicos, como sucede en la mayoría de las antologías (cf. Sabio Pinilla \& Ordóñez López 2012: 114-115). En el caso de Weissbort y Eysteinsson (2006) se crean secciones en las que se agrupa un determinado enfoque traductológico o un periodo determinado y se abordan de manera general algunas de las contribuciones más representativas, para así paliar o contrarrestar las inevitables omisiones.

Las referencias bibliográficas suelen presentarse al final de la obra, donde se incluyen tanto los textos recopilados como los trabajos citados en las secciones introductorias, cuando las hay. Además, salvo en el caso de Chesterman (1989), se proporcionan índices de materias (cf. Baker 2009), de nombres (cf. Weissbort \& Eysteinsson 2006; Baker 2009) o ambos de manera conjunta (cf. Venuti 2000, 2004).

En resumen, como rasgo distintivo de estas obras, podemos señalar su marcado componente didáctico, que se plasma en la inclusión de secciones de carácter introductorio, en la contextualización de los textos o en la sugerencia de lecturas y actividades complementarias. Presentamos ahora el manual de lecturas guiadas, que se situaría en la línea del modelo antológico anglosajón que acabamos de comentar.

\section{Propuesta de un manual de lecturas guiadas de historiografía}

Según lo expuesto, el manual proyectado estaría formado por un corpus de textos que recogerían las contribuciones de naturaleza historiográfica en lugar de documentos históricos propiamente dichos. Dentro de la tradición del reader, la obra cumpliría una función eminentemente pedagógica e incluiría una muestra representativa de la investigación histórica en traducción desarrollada en los últimos cuarenta años. Una obra de estas características debería entenderse como una aportación al conjunto de publicaciones que hoy día sirven de instrumentos analíticos y generales de la disciplina, pero con la particularidad de proporcionar una información contextualizada de 
la investigación historiográfica contemporánea a partir de los textos seleccionados. En este sentido, una de las funciones del manual es aportar a los estudiantes otro tipo de herramientas que les hagan ver la validez de la teoría en el proceso de aprendizaje de la traducción. Al mismo tiempo, el manual se concibe como obra de apoyo a los docentes y, por consiguiente, junto a los contenidos teóricos, incluiría materiales y actividades que permitan la explotación de los textos por parte de los profesores y animen a la reflexión de los alumnos.

Así pues, nos situamos en la perspectiva de la historiografía entendida como la disciplina que estudia el desarrollo de la investigación histórica o, dicho de otro modo, partimos de lo que se ha escrito sobre la investigación histórica en traducción y planteamos la necesidad de reunir en un volumen esa investigación con fines académicos y didácticos. Esta obra permitiría ofrecer una panorámica del estudio histórico en traducción, mostrando la evolución de una historiografía tradicional, de corte positivista, a otra historiografía posmoderna (o "postpositivista", cf. Tymoczko 2007), y ayudaría a reconstruir, de modo selectivo, el conjunto de conocimientos historiográficos que se corresponden con los enfoques traductológicos contemporáneos.

La elaboración de la obra, que estaría coordinada por los autores de este artículo, correría a cargo de un grupo de profesores y seguiría diferentes fases: recopilación del material bibliográfico, selección, traducción (como valor añadido a la función didáctica del manual) y revisión de los textos, redacción de entradas y preparación del material de apoyo. Los documentos se presentarían completos en la medida de lo posible o al menos con la suficiente extensión como para no desvirtuar su contenido.

Como punto de partida, defendemos en esta propuesta una selección de los textos más representativos de los investigadores contemporáneos con la que se dé cuenta de la evolución historiográfica y la pluralidad de tradiciones desde las que se formulan las cuestiones de teoría e historia. Así, serían representativos de esta idea aquellos textos que han servido para replantear críticamente aproximaciones anteriores en el campo de la investigación histórica en traducción e interpretación o aquellos otros que han propiciado el debate desde diferentes tradiciones traductológicas al incorporar perspectivas de otras disciplinas, planteando posicionamientos teóricos novedosos. Conviene señalar que las posturas teóricas no tienen una delimitación exacta por lo que muchos autores y textos pueden encuadrarse en más de un paradigma, escuela o modelo, dado el carácter interdisciplinar de nuestra disciplina; ahora bien, lo que nos interesa es mostrar las preferencias por las cuestiones teóricas e historiográficas al margen de una adscripción específica. Partiendo 
de esta premisa, estarían representados en el manual muchos de los autores citados en este artículo como, por ejemplo, D’hulst (cf. 1991, 1995, 2001, 2010), Lambert (cf. 1993) y Hermans (cf. 2004), que han sentado las bases metodológicas en torno a conceptos y métodos; no podemos olvidar las aportaciones desde los estudios filológicos, caso de Lépinette (cf. 1997), así como otros nombres indispensables por haber reparado en la importancia de los métodos del historiador en el estudio de la traducción como Delisle (cf. 1997-98) y Pym (cf. 1992, 1998). En esta línea, el manual debería de servir para fomentar un diálogo con las ciencias históricas pues, como han puesto de manifiesto algunos investigadores, la investigación histórica en traducción e interpretación puede ayudar a revisar la historia (cf. Payás 2006), ampliando los horizontes a las cuestiones de la globalización (cf. Cronin 2003), la identidad (cf. Cronin 2006), el poder (cf. Tymoczko \& Gentzler 2002) o el conflicto (cf. Baker 2002), y aplicando los estudios históricos a nuevos espacios como China (cf. Cheung 2009) o Hispanoamérica (cf. Bastin 2010-11). Tampoco faltarán traductólogos que realizan investigaciones plenamente interdisciplinares en sociología e historiografía (cf. Gouanvic 2006). En definitiva, se procurará dar cuenta de los textos más influyentes de las diferentes tradiciones traductológicas y posturas teóricas en relación con el tratamiento de la investigación histórica del pasado.

A la presentación del volumen, le seguiría una introducción a modo de estudio y análisis general del grueso de textos que, en principio, adoptaría el orden cronológico por año de publicación; los textos irían acompañados de notas biográficas de los autores para situarlos en sus respectivas tradiciones $y$, en su caso, de otras notas internas, además de un comentario general y de materiales didácticos; completaría la obra una bibliografía final, un glosario de conceptos básicos y un índice de nombres y temas.

Como hemos apuntado en este trabajo, el manual intentaría favorecer un conocimiento comparado del estudio histórico de la traducción a partir de textos representativos que reflejen un canon amplio, no solo eurocéntrico, y que sean relevantes. Asimismo, incluiría lecturas recomendadas con vistas a cubrir textos y autores no recogidos en la selección pero que se consideran importantes para completar la visión ofrecida o para desarrollar una determinada cuestión. El material de apoyo didáctico iría destinado al ámbito universitario e incluiría actividades de aplicación de los contenidos teóricos, preguntas para fomentar la discusión en clase o para elaborar posibles trabajos de ampliación o de profundización, con el objetivo de reforzar la vinculación entre teoría y práctica, así como un glosario de conceptos que incluya nociones historiográficas y traductológicas clave. 
En última instancia, la obra debería privilegiar una visión selectiva y al mismo tiempo variada del estudio histórico de la traducción donde estuvieran recogidas las diferentes tradiciones historiográficas con los nombres, textos, enfoques y tendencias que han ido consolidando críticamente este campo desde el último tercio del siglo veinte hasta nuestros días.

\section{Bibliografía}

BAKER, Mona. (2002) Translation and Conflict: A Narrative Account. London \& New York: Routledge.

BAKER, Mona (ed.) (2009) Critical Readings in Translation Studies. London \& New York: Routledge.

BAndia, Paul. (2006) "The Impact of Postmodern Discourse on the History of Translation." En: Bastin, George L. \& Paul F. Bandia (eds.) 2006. Charting the Future of Translation History. Ottawa: University of Ottawa Press. pp. 45-58.

BASTIN, George L. \& Paul F. Bandia (eds.) (2006) Charting the Future of Translation History. Ottawa: University of Ottawa Press.

BASTIN, George L. (2010-11) "La pertinencia de los estudios históricos sobre traducción en Hispanoamérica." Estudios Interdisciplinares de América Latina y el Caribe 21:1.

Chesterman, Andrew (ed.) (1989) Readings in Translation Theory. Helsinki: Oy Finn Lectura Ab.

Cheung, Martha. (2009) "Introduction. Chinese Discourses on Translation. Positions and Perspectives." The Translator 15:2 (Special Issue. Chinese Discourses on Translation). pp. 223-278.

Cronin, Michael. (2003) Translation and Globalization. London \& New York: Routledge.

Cronin, Michael. (2006) Translation and Identity. London \& New York: Routledge.

DELISLE, Jean. (1997-98) "Réflexions sur l'historiographie de la traduction et ses exigences scientifiques." Équivalences 26:2 y 27:1. pp. 21-43. [Versión en francés del artículo de 1996: "Reflexións sobre as esixencias científicas da historia da traducción." Viceversa 2. pp. 37-56].

DelisLE, Jean. (2008) "Historiographie, notions, sens, citations, CD-ROM." Mutatis, Mutandis 1:1. pp. 81-96.

D’hULST, Lieven. (1991) "Pourquoi et comment écrire l'histoire des théories de la traduction?" Actes du XII ${ }^{e}$ Congrès mondial de la FIT. Belgrado: Prevodilac. pp. 57-62.

D’HULST, Lieven. (1995) "Pour une historiographie des théories de la traduction: questions de méthode." TTR 8:1. pp. 13-33. 
D’HUlst, Lieven. (2001) "Why and How to Write Translation Histories?" En: Milton, John (ed.) 2001. Emerging Views on Translation History in Brazil. São Paulo: Crop. pp. 21-32.

D’HULST, Lieven. (2010) "Translation History." En: Gambier, Yves \& Luc van Doorslaer (eds.) 2010. Handbook of Translation Studies. Amsterdam \& Philadelphia: John Benjamins. pp. 397-405.

D’HULST, Lieven. (2011) Historiografía de la Traducción. Curso impartido dentro de las actividades del Seminario Permanente del Máster investigador en Traducción e Interpretación de la Facultad de Traducción e Interpretación de la Universidad de Granada, 25-28 de marzo.

ENRíQueZ ARANDA, María M. (2007) "La creación del canon a través de la retraducción en antologías: imagen romántica en el siglo XX.” En: Zaro Vera, Juan Jesús \& Francisco Ruiz Noguera (eds.) 2007. Retraducir: una nueva mirada. La retraducción de textos literarios y audiovisuales. Málaga: Gómez y Navarro Comunicación. pp. 117-134.

FERNÁNDEZ SÁNCHEZ, María Manuela. (2010) "Sobre el tratamiento de las fuentes en la historiografía posmoderna: archivos, fotografías y memorias de intérpretes en la Guerra Fría.” En: Rabadán, Rosa; Trinidad Guzmán \& Marisa Fernández (eds.) 2010. Lengua, traducción, recepción en honor de Julio César Santoyo. Language, Translation, Reception to Honor Julio César Santoyo. León: Universidad de León, Área de Publicaciones, vol. I. pp. 231-246.

FERNÁNDEZ SÁNCHEZ, María Manuela. (2012) “¿Por qué resulta tan prescindible el estudio de las teorías de la traducción? Una reflexión sobre los docentes como historiadores de la ciencia." En: Basich Peralta, Kora Evangelina; Sonia Acosta Domínguez; Ana Gabriela Guajardo Martínez Sotomayor; Miguel Ángel Lemus Cárdenas \& Ana Gabriela Rubio Moreno (coords.) 2012. Traduciendo culturas: más que un desafío. V Congreso Internacional de Traducción e Interpretación. Mexicali: Universidad Autónoma de Baja California. pp. 73-94.

FrAISSE, Emmanuel. (1997) Les anthologies en France. Paris: P.U.F.

GOUANVIC, Jean-Marc. (2006) "Au-delà de la pensée binaire en traductologie: esquisse d'une analyse sociologique des positions traductives en traduction littéraire." TTR: traduction, terminologie, rédaction 19:1. pp. 123-134.

GuillÉn, Claudio. [1985] (2005) Entre lo uno y lo diverso. Introducción a la Literatura Comparada (Ayer y hoy). Barcelona: Tusquets Editores (Marginales, 229).

Hermans, Theo. (1994) Translation in Systems. Descriptive and Systemic Approaches Explained. Manchester: St Jerome.

Hermans, Theo. (2004) "Metaphor and Image in the Discourse on Translation. A Historical Survey". En: Kittel, Harald; Armin Paul Frank; Norbert Greiner; Theo Hermans; Werner Koller; Jose Lambert \& Fritz Paul (eds.) 2004. Übersetzung. Translation. Traduction. Ein internationales Handbuch zur 
Übersetzungsforschung. An International Encyclopedia of Translation Studies. Encyclopédie internationale de la recherche sur la traduction. Berlin \& New York: Walter de Gruyter, vol. 1. pp. 118-128.

HolmES, James. (1972) "The Name and Nature of Translation Studies". En: Ovistgaard, Jacques et al. (eds.) 1972. International Congress of Applied Linguistics: Congress Abstracts (3rd, Copenhagen, 21-26 August 1972). Copenhagen: Ehrverskøkonomisk Forlag. Versión electrónica: < http://www.eric.ed.gov/ PDFS/ED074796.pdf>

Holmes, James. (1988) Translated! Papers on Literary Translation and Translation Studies. Amsterdam: Rodopi.

LamberT, Jose. (1993) "History, Historiography and the Discipline. A Programme." En: Gambier, Yves \& Jorma Tommola (eds.) 1993. Translation and Knowledge. Turku: University of Turku. pp. 3-25.

LAMBERT, Jose. (1995) "Translation, Systems and Research: The Contribution of Polysystem Studies to Translation Studies." TTR 8:1. pp. 105-152.

LEFEVERE, André. (1992) Translation, Rewriting, and the Manipulation of Literary Fame. London \& New York: Routledge.

LÉPINETTE, Brigitte. (1997) La historia de la traducción - Metodología. Apuntes bibliográficos. València: Universitat de València, Centro de Estudios sobre Comunicación Interlingüística e Intercultural (LynX Documentos de Trabajo, vol. 14).

LÉPINETTE, Brigitte. (2006) "García Garrosa, María José \& Francisco Lafarga, El discurso sobre la traducción en la España del siglo XVIII. Estudio y antología." Target 18:2. pp. 373-380.

LóPez GarCía, Dámaso. (2011) "Twitter-traducción”. En: Sáez, Daniel; Jorge Braga; Marta Abuín; Marta Guirao; Beatriz Soto \& Nava Maroto (ed.) 2011. Últimas tendencias en traducción e interpretación. Madrid: Iberoamericana. pp. 79-98.

MundaY, Jeremy. (2012) Introducing Translation Studies. London \& New York: Routledge ( $3^{\text {a }}$ edición).

PAYÁS, Gertrudis. (2006) "Lorsque l'histoire de la traduction sert a réviser l'histoire." TTR: traduction, terminologie, rédaction 19:2. pp. 15-36.

PYM, Anthony. (1992) "Shortcomings in the Historiography of Translation." Babel 38:4. pp. 221-235.

PYM, Anthony. (1998) Method in Translation History. Manchester: St Jerome.

PYM, Anthony. (2010) Exploring Translation Theories. London \& New York: Routledge.

Ruiz CaSANOva, José Francisco. (2007) Anthologos, poética de la antología poética. Madrid: Cátedra.

Sabio Pinilla, José Antonio \& Pilar Ordóñez López. (2012) Las antologías sobre la traducción en el ámbito peninsular. Análisis y estudio. Bern: Peter Lang (Col. 
Relaciones literarias en el ámbito hispánico: traducción, literatura y cultura, $6)$.

SANTOYO, Julio-César. (2009) La traducción medieval en la Península Ibérica (siglos III-XV). León: Universidad de León.

StÖRIG, Hans J. (1963) Das Problem des Übersetzens. Darmstadt: Wissenschaftliche Buchgessellschaft.

TyMoczKo, Maria \& Edwin Gentzler (eds.) (2002) Translation and Power. Amherst/Boston: University of Massachusetts Press.

Tyмoczko, Maria. (2007) Enlarging Translation, Empowering Translators. Manchester: St Jerome.

VENUTI, Lawrence (ed.) (2000) The Translation Studies Reader. London \& New York: Routledge.

Venuti, Lawrence (ed.) (2004) The Translation Studies Reader. London \& New York: Routledge [ $2^{\mathrm{a}}$ edición revisada].

Vidal Claramonte, Mª Carmen África. (1998) El futuro de la traducción: Últimas teorías, nuevas aplicaciones. Valencia: Institució Alfons el Magnánim.

WeIsSBORT, Daniel \& Astradur Eysteinsson (eds.) (2006) Translation. Theory and Practice. A Historical Reader. New York: Oxford University Press.

\section{NOTAS BIOGRÁFICAS / BIONOTES}

\section{Pilar Ordóñez López}

Pilar Ordóñez López es actualmente profesora contratada doctora en el Departamento de Traducción y Comunicación en la Universitat Jaume I y ha trabajado en diferentes universidades en el Reino Unido y Estados Unidos. Entre sus líneas de investigación se encuentra la historia y la teoría de la traducción, así como su didáctica a nivel universitario, y ha publicado diversos trabajos en estos ámbitos.

Pilar Ordóñez López is Lecturer in Translation Studies at the Universitat Jaume I in Castellón de la Plana, having previously taught at several universities in the United States and the United Kingdom. Her research areas include translation history and theory as well as translator training, and she has published extensively in those fields.

\section{José Antonio Sabio Pinilla}

José Antonio Sabio Pinilla es profesor titular de Traducción e Interpretación de la Universidad de Granada. Se dedica al estudio de las relaciones literarias entre España y Portugal desde la perspectiva de la historia y teoría de la 
traducción. Es autor o coautor de distintos volúmenes monográficos y ha editado diversas obras colectivas. Ha sido decano de la Facultad de Traducción e Interpretación de la Universidad de Granada.

José Antonio Sabio Pinilla is Senior Lecturer in Translation and Interpreting at the University of Granada. His research focuses mainly on the literary relations between Spain and Portugal from the perspective of the history and theory of translation. He has published and co-published several monographs and has edited various collective publications. He has been Dean of the Faculty of Translation and Interpreting of the University of Granada. 